\title{
Hydrodynamic Anisotropy Effects on Radiation-Mixed Convection Interaction in a Vertical Porous Channel
}

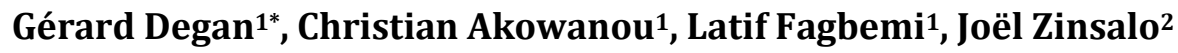 \\ ${ }^{1}$ Laboratoire d’Energétique et de Mécanique Appliquées (LEMA), Ecole Polytechnique d'Abomey-Calavi (EPAC), \\ Université d'Abomey-Calavi (UAC), Cotonou, Bénin \\ ${ }^{2}$ Department of Mechanical Engineering, Ecole de Technologie Supérieure, Montréal, Canada \\ Email:"djidjohoako@yahoo.fr, "ger_degan@yahoo.fr, latif@yahoo.fr, znisalo@yahoo.fr
}

Received 10 December 2015; accepted 11 January 2016; published 14 January 2016

Copyright (C) 2016 by authors and Scientific Research Publishing Inc.

This work is licensed under the Creative Commons Attribution International License (CC BY). http://creativecommons.org/licenses/by/4.0/

(c) (i) Open Access

\section{Abstract}

The effects of hydrodynamic anisotropy on the mixed-convection in a vertical porous channel heated on its plates with a thermal radiation are investigated analytically for fully developed flow regime. The porous medium is anisotropic in permeability whose principal axes are oriented in a direction that is oblique to the gravity. The generalized Brinkman-extended Darcy model which allows the no-slip boundary-condition on solid wall is used in the formulation of the problem. The flow reversal, the thermal radiation influence for natural, and forced convection are considered in the limiting cases for low and high porosity media. It was found that the anisotropic permeability ratio, the orientation angle of the principal axes of permeability and the radiation parameter affected significantly the flow regime and the heat transfer.

\section{Keywords}

Mixed-Convection, Thermal Radiation, Anisotropic Porous Medium, Flow Reversal, Heat Transfer

\section{Introduction}

Convection heat transfer in porous media is a fundamental importance in such technologies as geothermal exploitation, oil recovery, radioactive waste management, insulation of building and cold storage, drying processes, transpiration cooling, powder metallurgy, agricultural engineering, solidification and binary alloys, etc. It is also important to geophysics and environmental sciences. Much of this activity, both numerical and experimental, has

*Corresponding author.

How to cite this paper: Degan, G., Akowanou, C., Fagbemi, L. and Zinsalo, J. (2016) Hydrodynamic Anisotropy Effects on Radiation-Mixed Convection Interaction in a Vertical Porous Channel. Applied Mathematics, 7, 22-39.

http://dx.doi.org/10.4236/am.2016.71003 
been summarized by Nield and Bejan (1999) [1]. Moreover, a relevant recent work on the topic is done by Chen et al. (2000) [2].

Thermal radiation always exists and can strongly interact with convection in many situations of engineering interest. The convection heat transfer in a porous channel (or in an enclosed space) in the presence of thermal radiation continues to receive considerable attention because of its importance in many practical applications such as furnaces, combustion chambers, cooling towers, rocket engines and solar collectors. During the last decade, many experimental and numerical investigations on the phenomenon of the interaction of natural or mixed convection with thermal radiation in vertical porous channels or enclosures have been presented. Mahmud and Fraser (2003) [3] have examined analytically the effects of radiation heat transfer on magnetohydrodynamic mixed convection through a vertical channel packed with fluid saturated porous substances. Basing their analysis on the first and second laws of thermodynamics, these authors have given special attention to entropy generation characteristics and their dependency on the various governing parameters of the problem. Jbara et al. (2011) [4] have conducted a numerical study to investigate the effects of the radiation heat transfer on the transient natural convection in a vertical channel opened at both ends and filled with a fluid saturated porous medium. Taking into account the Rosseland approximation in the energy equation, they have showed that approximation is only valid for large Planck number values for temperature ratios closed to unity and/or for single scattering albedo near or equals to one. An unsteady natural convection magnetohydrodynamic flow of a rotating viscous electrically fluid in a vertical channel with radiation effects has been investigated by Chauban and Rastogi (2010) [5] who obtained analytical expressions for velocity and temperature fields and discussed the effects of radiation on the phenomenon. A transient study of coupled natural convection and radiation in a porous vertical channel using the finitevolume method has been conducted by Slimi et al. (2004) [6]. The results indicated that the controlling parameters of the problem have significant effects on the flow and the thermal fields and on the transient process of heating and cooling of the medium. They have demonstrated that the volumetric flow rate and the convected heat flux at the channel's exit increase when the Planck number is decreased and/or the optical thickness and the wall emissivity are increased.

Most of the existing theories and experimental investigations on the topic, are concerned isotropic porous media. However, in several applications the porous materials are anisotropic. Such porous media are in fact encountered in numerous systems in industry and nature. As examples, we can cite fibrous materials, biological materials, geological formations, and oil extraction. The inclusion of more physical realism in the matrix properties of the medium is important for the accurate modeling of the anisotropic media. Anisotropy is generally a consequence of a preferential orientation and asymmetric geometry of the grain or fibbers which constitute the porous medium. Despite its vast range of applications, convection in such anisotropic porous media has received relatively little attention.

Thermal convection in a porous medium with anisotropic permeability was first considered by Castinel and Combarnous (1974) [7] who conducted an experimental and theoretical investigation in a horizontal layer heated from below. Their results were extended by Epherre (1975) [8] and Kvernvold and Tyvand (1979) [9] who considered a more general type of anisotropy. Convection attributable to side-heating in a vertical layer has been considered by several authors. For example, the case of square cavity with homogeneous media that is both thermally and hydrodynamically anisotropic has been investigated analytically by Kimura et al. (1993) [10] and numerically by Ni and Beckermann (1991) [11]. The effect of both anisotropy of permeability and thermal conductivity on the overall Nusselt number was found to be equally significant. A few studies have also been concerned with the case when the principal axes of anisotropy of the porous structure are inclined with respect to the gravity force. For this situation, the onset of motion in a porous layer heated from below was predicted by Tyvand and Storesletten (1991) [12] and Zhang et al. (1993) [13]. It was demonstrated that the influence of the anisotropy orientation considerably modifies the stability limit. Convection heat transfer in a vertical cavity heated from the side with various thermal boundary conditions has been investigated by Zhang (1993) [14], Degan et al. (1995) [15] and Degan and Vasseur (1997, 2002) [16] [17]. It was demonstrated that the heat transfer was maximum when the principal axis with higher permeability is parallel to the vertical direction and minimum when it is perpendicular.

The contemporary trend in the field of heat transfer and thermal design is to apply a second law of thermodynamics analysis, and its design-related concept of entropy generation minimization (see Bejan (1996) [18]). Entropy generation is associated with thermodynamic irreversibility which is present in all heat transfer processes. Because of the abundance of publications on entropy, no attempt is made here to review the literature. 
The present work deals with an analytical study of coupled fluid flow and heat transfer by mixed convection and radiation in a vertical channel opened at both ends and filled with a fluid-saturated porous medium. The bounding walls of the channel are isothermal and gray. The effects of hydrodynamic anisotropy of the porous medium will be investigated, since the physical problem is of significant importance to many engineering-related applications.

\section{Mathematical Formulation}

The problem, under different considerations, concerns an optically thin and electrically conducting fluid flowing through a vertical channel opened at both ends and filled with a porous medium as shown by the physical model considered in Figure 1. The bounding walls of the channel are isothermal and kept at the same or different temperatures. Moreover, these bounding walls are submitted to the influence of radiative intensities $I_{1-2}$ and $I_{2-1}$ exerting reciprocally actions on the right and the left walls, respectively. The depth of the channel along the $z^{\prime}$ axis is assumed sufficiently long compared to other directions, $x^{\prime}$ being the direction of the flow and $y^{\prime}$ direction perpendicular to that latter. A fully developed, laminar and induced flow is maintained through this porous channel upward, so that the natural convection aids the forced flow. As considered in nature in fact, the porous medium is anisotropic in flow permeability, the permeabilities along the two principal axes of the porous matrix are denoted by $K_{1}$ and $K_{2}$. The anisotropy in flow permeability of the porous medium is then characterized by the permeability ratio $K^{*}=K_{1} / K_{2}$ and the orientation $\varphi$, defined as the angle between the horizontal direction and the principal axis with permeability $K_{2}$. The principal directions of the thermal conductivity are assumed to coincide to those of the coordinate axes. The saturating fluid is viscous, incompressible and assumed to be in local thermodynamic equilibrium with the solid matrix everywhere and its thermophysical properties are assumed constant, except for the density in the buoyancy term in the momentum equation.

Under the above approximations, the equations governing the conservation of mass, momentum (generalized Brinkman-extended Darcy's law) and energy can be written as follows (see [3] [17]):

$$
\begin{aligned}
& \nabla \cdot \boldsymbol{V}^{\prime}=0 \\
& \boldsymbol{V}^{\prime}=\frac{\overline{\bar{K}}}{\mu}\left(-\nabla p^{\prime}+\rho \boldsymbol{g}+\mu_{e f f} \nabla^{2} \boldsymbol{V}^{\prime}\right) \\
& \left(\rho c_{p}\right)_{f} \nabla \cdot\left(\boldsymbol{V}^{\prime} \cdot T^{\prime}-\alpha \nabla T^{\prime}\right)=-\nabla \cdot q^{R^{\prime}}
\end{aligned}
$$

where $\boldsymbol{V}^{\prime}$ is the superficial flow velocity, $p^{\prime}$ is the pressure, $\mu$ the dynamic viscosity, $\mu_{\text {eff }}$ apparent dynamic viscosity for Brinkman's model, $\left(\rho c_{p}\right)_{f}$ the heat capacity of the fluid, $\rho$ the density, $\alpha$ the thermal diffusivity, $T^{\prime}$ the temperature and $q^{R^{\prime}}$ represents the radiative heat flux. The symmetrical second-order permeability tensor $\overline{\bar{K}}$ is defined as:

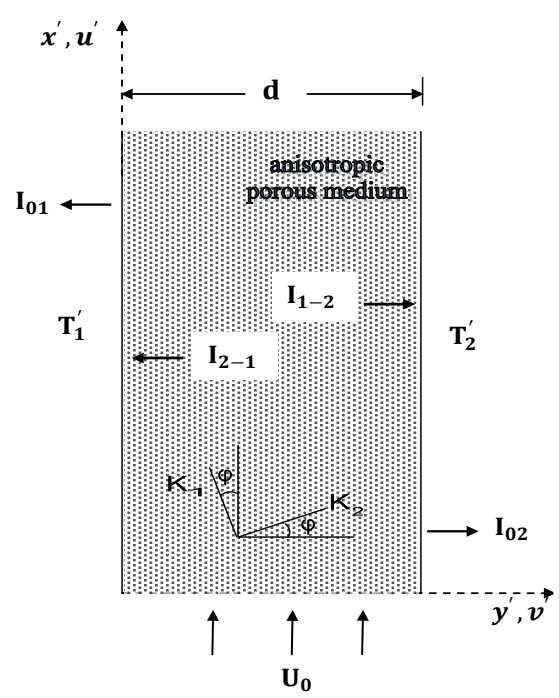

Figure 1. Physical model of the channel. 


$$
\overline{\bar{K}}=\left[\begin{array}{ll}
K_{1} \cos ^{2} \varphi+K_{2} \sin ^{2} \varphi & \left(K_{1}-K_{2}\right) \sin \varphi \cos \varphi \\
\left(K_{1}-K_{2}\right) \sin \varphi \cos \varphi & K_{1} \sin ^{2} \varphi+K_{2} \cos ^{2} \varphi
\end{array}\right]
$$

The energy Equation (3) takes into account the radiative heat flux term $\nabla \cdot q^{R^{\prime}}$. It is a non-linear term which will be evaluate to have approximate analytical solution for the present problem. Due to the fact that the radiative heat flux is normal to the bounding walls, $\partial q^{R^{\prime}} / \partial x^{\prime}=0$ and only the term $\partial q^{R^{\prime}} / \partial y^{\prime}=0$ will be considered in the energy equation, and for which an approximate mathematical model will be estimated. According to Arpaci et al. [19], one can write as follows:

$$
\frac{\partial q^{R^{\prime}}}{\partial y^{\prime}}=4 \varsigma E_{b}-\varsigma \int_{\Omega} I \mathrm{~d} \Omega
$$

where $\varsigma, \Omega$ and $E_{b}$ are the monochromatic absorption coefficient, solid angle, and blackbody emissive power, respectively. The second term at the right hand side of Equation (5) represents the effect of boundaries. Assuming hemispherical isotropy, and replacing actual intensity with two-stream intensities (outward and inward) as shown in Figure 1, the second term of the right hand side of Equation (5) can be written as $\Omega$ and $E_{b}$ are the monochromatic absorption coefficient, solid angle, and blackbody emissive power, respectively. The second term at the right hand side of Equation (5) represents the effect of boundaries. Assuming hemispherical isotropy, and replacing actual intensity with two-stream intensities (outward and inward) as shown in Figure 1, the second term of the right hand side of Equation (5) can be written as

$$
\int_{\Omega} I \mathrm{~d} \Omega \approx 2 \pi\left(I_{1-2}+I_{2-1}\right) .
$$

Considering that the absorption is negligible for a thin gas, $I_{1-2}$ and $I_{2-1}$ remain uniform across the thickness of the gas between the plates (see Figure 1). Consequently, $I_{1-2}$ and $I_{2-1}$ become identical. Approximating each stream as a relation between emission $\left(\varepsilon I_{0}\right)$ and reflexion $(\rho I), I_{1-2}$ and $I_{2-1}$ can be written as

$$
\left\{\begin{array}{l}
I_{1-2}=\varepsilon_{1} I_{01}+\rho_{1} I_{2-1} \\
I_{2-1}=\varepsilon_{2} I_{02}+\rho_{2} I_{1-2}
\end{array}\right.
$$

where $I_{1-2}$ and $I_{2-1}$ are the intensities of radiation, $\varepsilon_{1}$ and $\varepsilon_{2}$ the emissivities, and $\rho_{1}$ and $\rho_{2}$ the reflectivities on the left and the right bounding walls, respectively.

Solving the system of equations, Equations (7a) and (7b), the solutions for $I_{1-2}$ and $I_{2-1}$ yield

$$
\left.\begin{array}{c}
I_{1-2}=\frac{\varepsilon_{1} I_{01}+\rho_{1} \varepsilon_{2} I_{02}}{\left(1-\rho_{2} \rho_{1}\right)} \\
I_{2-1}=\frac{\varepsilon_{2} I_{02}+\rho_{2} \varepsilon_{1} I_{01}}{\left(1-\rho_{2} \rho_{1}\right)}
\end{array}\right\}
$$

Substituting results obtained for these intensities into Equation (6) and noting that $\rho+\varepsilon=1$ (the transmissivity being negligible in this study) and $\pi I=E_{b}$, one can have the following approximation

$$
\int_{\Omega} I \mathrm{~d} \Omega \approx 4 \frac{\left(\frac{1}{\varepsilon_{2}}-\frac{1}{2}\right) E_{b 1}+\left(\frac{1}{\varepsilon_{1}}-\frac{1}{2}\right) E_{b 2}}{\left(\frac{1}{\varepsilon_{2}}-\frac{1}{2}\right)+\left(\frac{1}{\varepsilon_{1}}-\frac{1}{2}\right)}
$$

where $E_{b 1}$ and $E_{b 2}$ are the monochromatic absorption coefficients on the left and the right walls, respectively.

Taking into account that the emissivities of the left and the right walls are identical, (i.e., $\varepsilon_{1}=\varepsilon_{2}$ ), the substitution of Equation (9) into Equation (5) yields

$$
\frac{\partial q^{R^{\prime}}}{\partial y^{\prime}}=4 \varsigma\left[E_{b}-\left(E_{b 1}+E_{b 2}\right) / 2\right] .
$$

In the above equation, $\left(E_{b 1}+E_{b 2}\right) / 2$ can be replaced by the mean emissive power $E_{b 0}$, such that the two 
terms, $E_{b}$ and $E_{b 0}$ can be further expressed as $\sigma T^{\prime 4}$ and $\sigma T_{0}^{\prime 4}$, where $\sigma$ is the Stefan-Boltzmann constant. These terms in $T^{\prime 4}$ appearing in the energy equation have to be linearized in order to obtain analytical solution. Expressing $T$ as a Taylor series about $T_{0}^{\prime}$ with the assumption of small $\left|T_{1}^{\prime}-T_{2}^{4}\right|$ yields

$$
T^{\prime 4}=T_{0}^{\prime 4}+4\left(T^{\prime}-T_{0}^{\prime}\right) T_{0}^{\prime 3}+\frac{12\left(T^{\prime}-T_{0}^{\prime}\right)^{2}}{2} T_{0}^{\prime 2}+\frac{24\left(T^{\prime}-T_{0}^{\prime}\right)^{3}}{6}+\frac{24\left(T^{\prime}-T_{0}^{\prime}\right)^{4}}{24} .
$$

Truncating the above series after the second term and using the definitions of $E_{b}$ and $E_{b 0}$, Equations (10) and (11) yield

$$
\frac{\partial q^{R^{\prime}}}{\partial y^{\prime}}=16 \varsigma \sigma T_{0}^{\prime 3}\left(T^{\prime}-T_{0}^{\prime}\right) .
$$

Introducing the Boussinesq approximation

$$
\rho=\rho_{0}\left[1-\beta\left(T^{\prime}-T_{0}^{\prime}\right)\right]
$$

and assuming that when the flow is fully developed in the channel, the axial ( $x^{\prime}$-direction) velocity depends only of the transverse coordinate $y^{\prime}$ (i.e., $u^{\prime}=u^{\prime}\left(y^{\prime}\right)$ ), and then from the continuity equation, the transverse velocity component must be zero (i.e., $\left.v^{\prime}=0\right)$ ). Moreover, accounting for uniform wall temperature, one gets $\partial T^{\prime} / \partial y^{\prime}=0$, and the governing equations (for steady convective flow) may be written in reduced form as

$$
\begin{aligned}
& \frac{\mathrm{d} u^{\prime}}{\mathrm{d} x^{\prime}}=0 \\
& K_{1} \lambda \frac{\mathrm{d}^{2} u^{\prime}}{\mathrm{d} y^{\prime 2}}-a u^{\prime}=\frac{K_{1}}{\mu} \frac{\partial p^{\prime}}{\partial x^{\prime}}-\frac{K_{1}}{v} g \beta\left(T^{\prime}-T_{0}^{\prime}\right) \\
& \frac{\mu}{K_{1}} c u^{\prime}-\frac{\partial p^{\prime}}{\partial y^{\prime}}=0 \\
& \frac{\mathrm{d}^{2} T^{\prime}}{\mathrm{d} y^{\prime 2}}-\frac{16 \sigma \varsigma}{k} T_{0}^{\prime 3}\left(T^{\prime}-T_{0}^{\prime}\right)=0
\end{aligned}
$$

where

$$
\begin{aligned}
& a=\cos ^{2} \varphi+K^{*} \sin ^{2} \varphi \\
& c=\left(1-K^{*}\right) \sin 2 \varphi, \quad K^{*}=K_{1} / K_{2} \\
& \lambda=\mu_{\text {eff }} / \mu .
\end{aligned}
$$

The hydrodynamic and thermal boundary conditions for the vertical channel are

$$
\begin{aligned}
& y^{\prime}=0: u^{\prime}=0 \quad T^{\prime}=T_{1}^{\prime} \\
& y^{\prime}=d: u^{\prime}=0 \quad T^{\prime}=T_{2}^{\prime} .
\end{aligned}
$$

Taking $d R e$ (and $d$ ), $U_{0}^{\prime}, \rho U_{0}^{\prime}$ and $\Delta T$ as respective dimensional scales for length $x^{\prime}$ (and $y^{\prime}$ ), velocities $u^{\prime}$ and $v^{\prime}$, pressure and temperature, the governing Equations (14) and (17) can be written in nondimensional form as

$$
\begin{aligned}
& \frac{\mathrm{d} u}{\mathrm{~d} x}=0 \\
& \frac{\mathrm{d}^{2} u}{\mathrm{~d} y^{2}}-\frac{\xi^{2}}{\lambda} u=\frac{1}{\lambda}\left(\frac{\partial p}{\partial x}-\frac{R a}{P e D a} T\right) \\
& \frac{c}{R e D a} u-\frac{\partial p}{\partial y}=0 \\
& \frac{\mathrm{d}^{2} T}{\mathrm{~d} y^{2}}-\tau \cdot P l \cdot T=0
\end{aligned}
$$


where

$$
\xi=\sqrt{\frac{a}{D a}}
$$

In the above equation, $R a=\frac{K_{1} g \beta \Delta T^{\prime} d}{v \alpha}$ is the Darcy-modified Rayleigh number based in permeability $K_{1}$, $R e=\frac{U_{0} d}{v}$ the Reynolds number, $D a=\frac{K_{1}}{d^{2}}$ the Darcy number, $P e=\frac{U_{0} d}{\alpha}=R e \cdot \operatorname{Pr}$ the Peclet number, $\operatorname{Pr}=\frac{v}{\alpha}$ the Prandtl number, $P l=\frac{16 \sigma T_{0}^{\prime 3} d}{k}$ the Planck number and $\tau=\varsigma d$ the dimensionless optical thickness. The dimensionless temperature $T$ is defined as $T=\frac{T^{\prime}-T_{0}^{\prime}}{\Delta T^{\prime}}$ where $T_{0}^{\prime}$ is the reference temperature. For the reference temperature difference, $\Delta T$, assuming that $T_{2}^{\prime}>T_{0}^{\prime}$, one can chose $\Delta T=T_{2}^{\prime}-T_{0}^{\prime}$.

The boundary conditions, Equations (19) and (20) become

$$
\begin{array}{ll}
y=0: u=0 & T=\theta \\
y=1: u=0 & T=1
\end{array}
$$

where $\theta=\left(T_{1}^{\prime}-T_{0}^{\prime}\right) / \Delta T$.

From the dimensionless Equations (21) and (24) and the boundary conditions Equations (26) and (27), it is seen that the present problem is governed by eight dimensionless parameters, namely $\mathrm{Ra}, \mathrm{Da}, \mathrm{Re}, \mathrm{Pe}, \lambda, \mathrm{Pl}$, $\tau$ and $\theta$. However, in the present study, the value of $\mu_{e f f}$ in Brinkman's extension is taken, as a first approximation, equal to $\mu$ (i.e., $\lambda=1$ ).

\section{Analysis}

\subsection{Fully Developed Flow Solution}

Using the boundary conditions for the temperature, Equation (24) can be integrated to give the following fully developed temperature profile

$$
T(y)=\frac{1-\theta \cosh \left(R_{d}\right)}{\sinh \left(R_{d}\right)} \sinh \left(R_{d} y\right)+\theta \cosh \left(R_{d} y\right) .
$$

By substituting Equation (28) into Equation (22), and using the boundary conditions, Equations (26) and (27), the velocity profile is obtained as follows

$$
\begin{aligned}
u(y)= & \frac{\gamma}{\xi^{2}}[\cosh (\xi y)+A \sinh (\xi y)-1]+\frac{R a}{P e D a} \frac{1}{R_{d}^{2}-\xi^{2}}\left\{\theta\left[\cosh (\xi y)-\cosh \left(R_{d}\right)\right]\right. \\
& \left.+B \sinh \left(R_{d} y\right)-C \sinh (\xi y)\right\}
\end{aligned}
$$

where

$$
\left.\begin{array}{l}
A=\frac{1}{\sinh (\xi)}-\operatorname{coth}(\xi) \\
B=\theta \operatorname{coth}\left(R_{d}\right)-\frac{1}{\sinh \left(R_{d}\right)} \\
C=\theta \operatorname{coth}(\xi)-\frac{1}{\sinh (\xi)}
\end{array}\right\}
$$

In the above expression of the distribution of the velocity, the parameter $\gamma$ still remains to be evaluated. Substituting of Equation (29) into the continuity Equation (21) then gives directly the pressure variation as 


$$
\gamma=\frac{\partial p}{\partial x}=\frac{\xi^{3} \sinh (\xi)}{2 \cosh (\xi)-\xi \sinh (\xi)-2}\left\{1+\frac{R a}{P e D a} \frac{(1+\theta)}{\left(R_{d}^{2}-\xi^{2}\right)}\left[\frac{\cosh \left(R_{d}\right)-1}{R_{d} \sinh \left(R_{d}\right)}-\frac{\cosh (\xi)-1}{\xi \sinh (\xi)}\right]\right\} .
$$

It is noticed that when $R_{d} \rightarrow 0$, one can find that

$$
\gamma=\frac{\partial p}{\partial x}=\frac{\xi^{3} \sinh (\xi)}{2 \cosh (\xi)-\xi \sinh (\xi)-2}+\frac{(1+\theta)}{2} \frac{R a}{P e D a} .
$$

This result is in agreement with that which has been found by Degan and Vasseur [17].

The wall friction is defined by the following expression

$$
f R e=\frac{\tau_{w}}{\frac{1}{2} \rho u^{\prime 2}} \frac{u^{\prime} d}{v}= \pm\left. 2 \frac{\mathrm{d} u}{\mathrm{~d} y}\right|_{y=0,1}
$$

where the plus and minus signs correspond to the left and the right walls. Hence, on the left wall the friction is expressed as follows

$$
f R e=2\left[\frac{A \gamma}{\xi}+\frac{R a}{P e D a} \frac{1}{\left(R_{d}^{2}-\xi^{2}\right)}\left(R_{d} B-\xi C\right)\right]
$$

while, on the right wall, for the friction one can have

$$
\begin{aligned}
f R e= & -2 \frac{\gamma}{\xi}[\sinh (\xi)+A \cosh (\xi)]-2 \frac{R a}{P e D a} \frac{1}{\left(R_{d}^{2}-\xi^{2}\right)}\left\{\theta\left[\xi \sinh (\xi)-R_{d} \sinh \left(R_{d}\right)\right]\right. \\
& \left.+R_{d} B \cosh \left(R_{d}\right)-C \xi \cosh (\xi)\right\}
\end{aligned}
$$

such that the average friction defined by $\overline{f R e}=\left[\left.(f R e)\right|_{y=0}+\left.(f R e)\right|_{y=1}\right] / 2$ is expressed as follows

$$
\overline{f R e}=\frac{1}{2 \cosh (\xi)-\xi \sinh (\xi)-2}\left\{2 \xi^{2}[1-\cosh (\xi)]+\frac{R a}{P e D a} \frac{(1+\theta)}{\left(R_{d}^{2}-\xi^{2}\right)}\left(D \frac{\cosh (\xi)-1}{\xi \sinh (\xi)}+E \frac{\cosh \left(R_{d}\right)-1}{R_{d} \sinh \left(R_{d}\right)}\right)\right\}
$$

where

$$
\left.\begin{array}{l}
D=2\left(\xi^{2}-1\right)[\cosh (\xi)-1]+\xi \sinh (\xi) \\
E=2\left(R_{d}^{2}-\xi^{2}\right)[\cosh (\xi)-1]-\xi R_{d}^{2} \sinh (\xi)
\end{array}\right\}
$$

Concerning theory of fully developed confined convection including flow reversal in vertical channels, when buoyancy effects are increased (i.e., when the heat flux is increased), the fluid will accelerate near the walls. Then, mass conservation requires that the fluid decelerates in the center of the channel. Consequently, if buoyancy effects are strong enough, a minimum will form in the velocity distribution at the channel centerline. For even stronger buoyancy effects, a flow reversal will form at the channel centerline. In the case of aiding mixed convection through the porous channel, when a reverse flow occurs, the relatively lower velocity negative flow passes along side the cold wall hence carries a lower level of thermal energy. Since a net dimensionless mass flow is fixed, an equal quantity of fluid is added to the fluid flowing in the positive (upward) direction in this fluid flows adjacent to the hot wall thereby carrying a larger amount of energy.

Following Aung and Worku [20] and many authors, it is possible to deduce an expression or a criterion by which to predict whether flow reversal occurs. It is seen that, when $\theta<1$, the occurrence of flow reversal is given by the condition

$$
\left.\frac{\mathrm{d} u}{\mathrm{~d} y}\right|_{y=0}<0 .
$$

Applying Equation (29), the above condition translates into 


$$
\frac{R a}{P e D a}(1-\theta)>\frac{F_{R_{d}}(\xi)}{G_{R_{d}}(\xi)+H_{R_{d}}(\xi)+K_{R_{d}}(\xi)}
$$

where

$$
\left.\begin{array}{l}
F_{R_{d}}(\xi)=\xi^{2} R_{d}\left(\xi^{2}-R_{d}^{2}\right)[1-\cosh (\xi)] \sinh \left(R_{d}\right) \sinh (\xi) \\
G_{R_{d}}(\xi)=\xi^{2} \sinh (\xi)\left[\cosh \left(R_{d}\right)-1\right][1-\cosh (\xi)] \\
H_{R_{d}}(\xi)=\xi R_{d} \sinh \left(R_{d}\right)[\cosh (\xi)-1]^{2} \\
K_{R_{d}}(\xi)=R_{d}[2 \cosh (\xi)-\xi \sinh (\xi)-2]\left[\xi \sinh \left(R_{d}\right)-R_{d} \sinh (\xi)\right]
\end{array}\right\}
$$

Accordingly, one can deduce the flow reversal function $\left(F R F_{R_{d}}(\xi)\right)$ as

$$
F R F_{R_{d}}(\xi)=\frac{F_{R_{d}}(\xi)}{G_{R_{d}}(\xi)+H_{R_{d}}(\xi)+K_{R_{d}}(\xi)} .
$$

Two cases are of interest, one with $\xi \ll 1$ and the other with $\xi \gg 1$.

- Case with $\xi \ll 1$ (i.e., $a \ll D a$ ): This case corresponds to a weaker anisotropic porous medium situation for which the resistance resulting from the boundary effects is predominant with respect to that due to the solid matrix, as $D a \rightarrow \infty$ when $\xi \rightarrow 0$. This situation approaches to the fluid medium case in which the anisotropic effects of the porous medium are irrelevant. As a result, the pressure gradient, from Equation (31), is given by

$$
-\gamma=\left(-\frac{\partial p}{\partial x}\right) \approx 12\left(1+\frac{\xi^{2}}{6}\right)\left\{1-\frac{(1+\theta)}{\left(R_{d}^{2}-\xi^{2}\right)} \frac{R a}{P e D a}\left[\frac{\cosh \left(R_{d}\right)-1}{R_{d} \sinh \left(R_{d}\right)}-\frac{1}{2}\left(1-\frac{\xi^{2}}{12}\right)\right]\right\} .
$$

Consequently, as $\xi$ goes to zero, the limit of the pressure gradient is written as

$$
\lim _{\xi \rightarrow 0}\left(-\frac{\partial p}{\partial x}\right)=12\left\{1-\frac{(1+\theta)}{R_{d}^{2}} \frac{R a}{P e D a}\left[\frac{\cosh \left(R_{d}\right)-1}{R_{d} \sinh \left(R_{d}\right)}-\frac{1}{2}\right]\right\} .
$$

Then, the velocity profile, Equation (29) and its limit, as $\xi$ goes to zero, become as follows

$$
\begin{aligned}
u(y) \approx & \left(6 y-6 y^{2}\right)\left(1+\frac{\xi^{2}}{6}\right) \\
& +\frac{R a}{P e D a}\left\{\frac{(\theta+1)}{4}\left(y-y^{2}\right)-\frac{\theta}{2} y^{2}+\frac{1}{R_{d}^{2}}\left(\frac{\left(\theta \cosh \left(R_{d}\right)-1\right)}{\sinh \left(R_{d}\right)} \sinh \left(R_{d} y\right)-(\theta-1) y\right)\right\}
\end{aligned}
$$

and

$$
\begin{aligned}
\lim _{\xi \rightarrow 0} u= & \left(6 y-6 y^{2}\right) \\
& +\frac{R a}{P e D a}\left\{\frac{(\theta+1)}{4}\left(y-y^{2}\right)-\frac{\theta}{2} y^{2}+\frac{1}{R_{d}^{2}}\left(\frac{\left(\theta \cosh \left(R_{d}\right)-1\right)}{\sinh \left(R_{d}\right)} \sinh \left(R_{d} y\right)-(\theta-1) y\right)\right\} .
\end{aligned}
$$

The average friction and its limit, as $\xi$ goes to zero, are

$$
\overline{f R e} \approx 12\left(1+\frac{\xi^{2}}{12}\right)-\frac{12(1+\theta)}{15} \frac{15-\xi^{2}}{\left(R_{d}^{2}-\xi^{2}\right)}\left[\frac{1}{2}-\frac{\cosh \left(R_{d}\right)-1}{R_{d} \sinh \left(R_{d}\right)}\right]
$$

and

$$
\lim _{\xi \rightarrow 0} \overline{f R e}=12\left\{1-\frac{(1+\theta)}{R_{d}^{2}}\left[\frac{1}{2}-\frac{\cosh \left(R_{d}\right)-1}{R_{d} \sinh \left(R_{d}\right)}\right]\right\} .
$$


Also, the flow reversal function, Equation (41) and its limit can be written as

$$
F R F_{R_{d}}(\xi) \approx \frac{6 R_{d}\left(R_{d}^{2}-\xi^{2}\right)\left(1+\frac{\xi^{2}}{4}+\frac{\xi^{4}}{40}\right) \sinh \left(R_{d}\right)}{R_{d}\left[2 \sinh \left(R_{d}\right)+R_{d}\right]-6\left[\cosh \left(R_{d}\right)-1\right]}
$$

and

$$
\lim _{\xi \rightarrow 0} F R F_{R_{d}}(\xi)=\frac{6 R_{d}^{3} \sinh \left(R_{d}\right)}{R_{d}\left[2 \sinh \left(R_{d}\right)+R_{d}\right]-6\left[\cosh \left(R_{d}\right)-1\right]} .
$$

Consequently, as $R_{d}$ goes to zero, applying Equation (49), the function of the flow reversal function's limit and its limit

$$
\begin{aligned}
& F R F_{R_{d}}\left(R_{d}\right)=72\left(1+\frac{R_{d}^{2}}{6}\right)\left(1-\frac{R_{d}^{2}}{10}\right) \\
& \lim _{\xi \rightarrow 0} F R F_{R_{d}}\left(R_{d}\right)=72 .
\end{aligned}
$$

Similar results has been obtained by Degan and Vasseur [17]. The same remark is also made when comparing the result above with that one obtained by Aung and Worku [20] for the situation corresponding to a pure fluid medium in absence of radiation condition imposed on the walls.

- Case with $\xi \gg 1$ (i.e., $a \gg D a$ ): This case corresponds to a low porosity medium (the Darcy medium situation) in which the anisotropic effects are predominant, as $D a \rightarrow 0$ when $\xi \rightarrow \infty$. In this situation, one can obtain the following results for the velocity profile and its limit

$$
\begin{aligned}
u(y) \approx & \frac{\xi}{\xi-2}\left(1-\frac{\mathrm{e}^{-\xi y}}{2}-\mathrm{e}^{-\xi(1-y)}\right)\left\{1+\frac{R a}{P e D a} \frac{(1+\theta)}{\left(R_{d}^{2}-\xi^{2}\right)}\left(\frac{\left(\cosh \left(R_{d}\right)-1\right)}{R_{d} \sinh \left(R_{d}\right)}-\frac{1}{\xi}\right)\right\} \\
& +\frac{R a}{P e D a} \frac{1}{R_{d}^{2}-\xi^{2}}\left\{\theta\left(\frac{\mathrm{e}^{-\xi y}}{2}-\cosh \left(R_{d} y\right) \sinh \left(R_{d} y\right)\right)-\left(\mathrm{e}^{-\xi(1-y)}+\frac{\sinh \left(R_{d} y\right)}{\sinh \left(R_{d}\right)}\right)\right\}
\end{aligned}
$$

and

$$
\lim _{\xi \rightarrow 0} u(y)=1-\frac{\mathrm{e}^{-\xi y}}{2}-\mathrm{e}^{-\xi(1-y)} .
$$

Also, in this limiting situation, the pressure gradient, the average friction and the flow reversal function are expressed as follows

$$
\begin{gathered}
\gamma=\frac{\xi^{3}}{2-\xi}\left\{1+\frac{R a}{P e D a} \frac{(1+\theta)}{\left(R_{d}^{2}-\xi^{2}\right)}\left[\frac{\cosh \left(R_{d}\right)-1}{R_{d} \sinh \left(R_{d}\right)}-\frac{1}{\xi}\right]\right\} \\
\overline{f R e} \approx \frac{2 \xi^{2}}{\xi-2}
\end{gathered}
$$

and

$$
\operatorname{FRF}_{R_{d}}(\xi) \approx \frac{2 \xi^{4}}{(\xi-2)^{2}}
$$

\subsection{Limiting Case of Forced Convection Solution}

The limiting case of forced convection solution is obtained by setting $(R a / P e)=0$ in Equation (29). Then the velocity distribution is given by 


$$
u=\frac{\xi \sinh (\xi)}{2 \cosh (\xi)-\xi \sinh (\xi)-2}\left\{\frac{\sinh (\xi y)-\sinh [\xi(y-1)]}{\sinh (\xi)}-1\right\} .
$$

Here, the pressure variation is determined by the following expression

$$
-\frac{\partial p}{\partial x}=-\frac{\xi^{3} \sinh (\xi)}{2 \cosh (\xi)-\xi \sinh (\xi)-2} .
$$

The results presented above will be specified for two cases of interest ( $\xi \ll 1$ and $\xi \gg 1$ ).

- $\xi \ll 1$ : For this case, the velocity profile is expressed as

$$
u=\left(1+\frac{\xi^{2}}{6}\right)\left(6 y-6 y^{2}\right) .
$$

The pressure gradient is written

$$
-\frac{\partial p}{\partial x}=12\left(1+\frac{\xi^{2}}{6}\right)
$$

- $\quad \xi \gg 1$ : In this situation, one can obtain the following results for the velocity profile

$$
u=\frac{\xi}{\xi-2}\left(1-\frac{\mathrm{e}^{-\xi y}}{2}-\mathrm{e}^{-\xi(1-y)}\right)
$$

and for the pressure gradient

$$
-\frac{\partial p}{\partial x}=-\frac{\xi^{3}}{2-\xi} .
$$

\subsection{Limiting Case of Natural Convection Solution}

This important limiting case will be studied by setting $\gamma=\partial p / \partial x=0$. For natural convection, the entrance velocity cannot be specified. In this case, new dimensionless variables should be used as

$$
x=\frac{x^{\prime}}{d R e}, \quad y=\frac{y^{\prime}}{d}, \quad u=\frac{u^{\prime} d}{v R a}, T=\frac{T^{\prime}-T_{0}^{\prime}}{T_{2}^{\prime}-T_{0}^{\prime}}
$$

and, as $\lambda=1$ in this study, Equation (22) can be rewritten as follow

$$
\frac{\mathrm{d}^{2} u}{\mathrm{~d} y^{2}}-\zeta^{2} u=-\frac{1}{D a} T .
$$

The solution for the velocity distribution may be written in terms of variables utilized herein, giving

$$
u=\frac{1}{D a} \frac{1}{\left(R_{d}^{2}-\xi^{2}\right)}\left\{\theta\left[\cosh (\xi y)-\cosh \left(R_{d}\right)\right]+B \sinh \left(R_{d} y\right)-C \sinh (\xi y)\right\} .
$$

In the above velocity profile, the corresponding expressions to $B$ and $C$ are those indicated in Equation (30).

Taking $R_{d} \rightarrow 0$, one obtains similar profile to that one obtained by Degan and Vasseur [18].

The volume flow rate b per unit channel width is defined as $m=\int_{0}^{d} u^{\prime} d \mathrm{~d} y^{\prime}$ which becomes in dimensionless form as

$$
M=\frac{m P r}{d v R a}=\int_{0}^{1} u \mathrm{~d} y
$$

that must be calculated by the expression

$$
M=\frac{1}{D a} \frac{(1+\theta)}{\left(R_{d}^{2}-\xi^{2}\right)}\left\{\frac{\cosh (\xi)-1}{\xi \sinh (\xi)}-\frac{\left[\cosh \left(R_{d}\right)-1\right]}{R_{d} \sinh \left(R_{d}\right)}\right\} .
$$


When $R_{d} \rightarrow 0$, Equation (67) gives similar expression to that one obtained by Degan and Vasseur [17] corresponding to the case studied here when the radiation effect on the side walls is not taken into account.

The total heat absorbed by the fluid in traversing the channel is

$$
Q^{\prime}=\rho c_{p} \int_{0}^{d} u^{\prime}\left(T^{\prime}-T_{0}^{\prime}\right) \mathrm{d} y^{\prime}+\int_{0}^{d} q^{R^{\prime}} \mathrm{d} y^{\prime} .
$$

Writting $Q^{\prime}$ in its dimensionless form and using Equations (28) and (65), the total heat absorbed is obtained as

$$
\begin{aligned}
Q= & \int_{0}^{1} u T \mathrm{~d} y+\int_{0}^{1} q^{R} \mathrm{~d} y \\
= & \frac{1}{2 D a} \frac{1}{\left(R_{d}^{2}-\xi^{2}\right)}\left\{B \theta\left(\frac{\cosh \left(2 R_{d}\right)-1}{R_{d}}-\frac{\cosh \left(R_{d}-\xi\right)}{\left(R_{d}-\xi\right)}-\frac{\cosh \left(R_{d}+\xi\right)}{\left(R_{d}+\xi\right)}+\frac{2 R_{d}}{\left(R_{d}^{2}-\xi^{2}\right)}\right)\right. \\
& +B C\left(\frac{\sinh \left(R_{d}+\xi\right)}{\left(R_{d}+\xi\right)}-\frac{\sinh \left(R_{d}-\xi\right)}{\left(R_{d}-\xi\right)}\right)-B^{2}\left(\frac{\sinh \left(2 R_{d}\right)-\left(2 R_{d}\right)}{2 R_{d}}\right) \\
& +\theta^{2}\left(\frac{\sinh \left(R_{d}+\xi\right)}{\left(R_{d}+\xi\right)}+\frac{\sinh \left(R_{d}-\xi\right)}{\left(R_{d}-\xi\right)}-\frac{\sinh \left(2 R_{d}\right)+\left(2 R_{d}\right)}{2 R_{d}}\right) \\
& \left.-C \theta\left(\frac{\cosh \left(R_{d}+\xi\right)}{\left(R_{d}+\xi\right)}-\frac{\cosh \left(R_{d}-\xi\right)}{\left(R_{d}-\xi\right)}+\frac{2 \xi}{\left(R_{d}^{2}-\xi^{2}\right)}\right)\right\}-\frac{4(1-\theta)}{3 \tau T_{0}}
\end{aligned}
$$

where $B$ and $C$ are the corresponding expressions indicated in Equation (30).

An average Nusselt number may be defined as

$$
N u=\frac{Q^{\prime}}{k(2 d)\left(T_{2}^{\prime}-T_{0}^{\prime}\right) / d}=\frac{1}{2} R a Q
$$

where $R a\left(=\left(K_{1} g \beta \Delta T^{\prime} d / v \alpha\right)\right)$ is the Darcy-modified Rayleigh number and $Q$ the total heat absorbed, calculated in Equation (69).

The flow reversal condition for the limiting case of natural convection is

$$
\left.\frac{\mathrm{d} u}{\mathrm{~d} y}\right|_{y=0}=\frac{1}{D a} \frac{1}{\left(R_{d}^{2}-\xi^{2}\right)}\left\{\theta\left[\frac{R_{d} \cosh \left(R_{d}\right)}{\sinh \left(R_{d}\right)}-\frac{\xi \cosh (\xi)}{\sinh (\xi)}\right]-\frac{R_{d}}{\sinh \left(R_{d}\right)}+\frac{\xi}{\sinh (\xi)}\right\}<0
$$

such that the flow reversal criterion becomes

$$
\theta<\frac{R_{d} \sinh (\xi)-\xi \sinh \left(R_{d}\right)}{\xi \cosh (\xi) \sinh \left(R_{d}\right)-R_{d} \cosh \left(R_{d}\right) \sinh (\xi)} .
$$

Taking $R_{d} \rightarrow 0$, one obtains from Equation (72), similar flow reversal occurrence criterion to that one obtained by Degan and Vasseur [17].

We notice here two cases of interest, the first one with $\xi \ll 1$ and the second with $\xi \gg 1$.

- $\xi \ll 1$ : The velocity and the flow rate and their limiting values as $\xi$ goes to zero are calculated as

$$
\begin{gathered}
u=\frac{1}{D a} \frac{1}{\left(R_{d}^{2}-\xi^{2}\right)}\left\{\theta\left[\left(1+\frac{1}{2} \xi^{2} y^{2}\right)-\cosh \left(R_{d} y\right)\right]+\left(\frac{\theta \cosh \left(R_{d}\right)-1}{\sinh \left(R_{d}\right)}\right) \sinh \left(R_{d} y\right)\right. \\
\left.-\left\{(\theta-1) y-(\theta-1) \xi^{2} \frac{y^{3}}{6}-(2 \theta+1) \xi^{2} \frac{y}{6}+\frac{\xi^{4}}{36}\left[y^{3}-\theta(y-1)^{3}\right]\right\}\right\} \\
M=\frac{1}{D a} \frac{(1+\theta)}{\left(R_{d}^{2}-\xi^{2}\right)}\left\{\frac{1}{2}\left(1-\frac{\xi^{2}}{12}\right)-\frac{\left[\cosh \left(R_{d}\right)-1\right]}{R_{d} \sinh \left(R_{d}\right)}\right\}
\end{gathered}
$$


and

$$
\begin{gathered}
\lim _{\xi \rightarrow 0} u=\frac{1}{D a}\left\{\theta\left[1-\cosh \left(R_{d} y\right)\right]+\left(\frac{\theta \cosh \left(R_{d}\right)-1}{\sinh \left(R_{d}\right)}\right) \sinh \left(R_{d} y\right)-\left\{(\theta-1) y-(\theta-1) \frac{y^{3}}{6}+\theta \frac{y^{2}}{2}+(2 \theta+1) \frac{y}{6}\right\}\right\}(75) \\
\lim _{\xi \rightarrow 0} M=\frac{1}{D a} \frac{(1+\theta)}{\left(R_{d}^{2}\right)}\left[\frac{1}{2}-\frac{\left[\cosh \left(R_{d}\right)-1\right]}{R_{d} \sinh \left(R_{d}\right)}\right]
\end{gathered}
$$

- $\xi \gg 1$ :It is found in this case that, the velocity and the flow volume rate and the average Nusselt number are expressed as follows

$$
\begin{gathered}
u=\frac{1}{D a} \frac{1}{\left(R_{d}^{2}-\xi^{2}\right)}\left[\theta \mathrm{e}^{\xi y}+\mathrm{e}^{-\xi(y+1)}-\frac{\theta \sinh \theta\left[R_{d}(1-y)\right]+\sinh \left(R_{d} y\right)}{\sinh \left(R_{d}\right)}\right] \\
M=\frac{1}{D a} \frac{(1+\theta)}{\left(R_{d}^{2}-\xi^{2}\right)}\left[\frac{1}{\xi}\left(1-2 \mathrm{e}^{-\xi}\right)-\frac{\left[\cosh \left(R_{d}\right)-1\right]}{R_{d} \sinh \left(R_{d}\right)}\right] \\
N u=-\frac{1}{4 a} R_{a}\left\{B \theta\left(\frac{\cosh \left(2 R_{d}\right)-1}{R_{d}}\right)-B^{2}\left(\frac{\sinh \left(2 R_{d}\right)-\left(2 R_{d}\right)}{2 R_{d}}\right)-\theta^{2}\left(\frac{\sinh \left(2 R_{d}\right)+\left(2 R_{d}\right)}{2 R_{d}}\right)\right\} \\
-\frac{4(1-\theta)}{3 \tau T_{0}}
\end{gathered}
$$

It is noticed that when $R_{d} \rightarrow 0$, one can find that

$$
N u=\frac{1}{2 a} R_{a}\left[\frac{(1-\theta)^{2}}{3}+\theta\right]-\frac{4(1-\theta)}{3 \tau T_{0}} .
$$

Similar result has been found by Degan and Vasseur [17] for analogous situation when the thermal radiation effect is not taking into account.

\section{Results and Discussion}

The effects of varying $R_{d}$, the radiation parameter, $R_{d}$ and $\varphi$, the anisotropic parameters on the velocity profile for the case of mixed convection using Equation (29) are presented in Figures 2-4 for $R a / P e=5$, $\theta=0.5$ and $D a=0.01$.

In Figure 2, the radiation parameter $R_{d}$ affects the flow by promoting the convective circulation within the channel near the hot wall. The inverse behavior is obtained when $R_{d} \rightarrow 0$ for which a reverse flow is observed as the fluid near the cold wall sinks and the velocity decreases more and more. Such a conclusion has also been made by Degan and Vasseur [17] when studying mixed-convection through a vertical anisotropic porous channel with oblique principal axes without radiation effect. Aung and Worku [20] explained that, the reverse motion which occurs in the flow field is due to the fact that the ratio of wall temperature differences $\theta$ is less than unity.

From Figure 3 and Figure 4, when $R_{d}=2, R a / P e=5, \theta=0.5$ and $D a=0.01$, it is observed that near the hot wall, the velocity increased as the anisotropic parameters of the porous medium $K^{*}$ and $\varphi$ are made higher. Moreover, the curves show that the peak value of velocity increases rapidly near the hot wall as $\varphi$ or $K^{*}$ increases. Contrary to the following behavior, increasing values of these parameters contribute to slow the convective circulation along the warm wall as it is shown in Figure 3 when $\varphi \rightarrow 0^{\circ}$ for $K^{*}<1\left(K^{*}=0.1\right)$, all curves sink in the neighborhood of the cold wall but the reversal flow is absent. We concluded that small values of anisotropic parameters of the porous medium contribute to stabilize the fluid motion.

Figure 5 shows the dimensionless temperature profile for mixed convection when $\theta=0.5$ for various values of the radiation parameter $R_{d}$. Curves plotted in Figure 5 reveal that the temperature decreases with an increase of $R_{d}$. When $R_{d}=0$, the temperature distribution is a function only of the ratio of wall temperature differences $\theta$ as predited by Aung and Worku [21]. Moreover, for all values of $R_{d}>0$, the temperature is lower than that of the absence of thermal radiation. One can deduce that, the thermal radiation contributes to reduce the temperature 


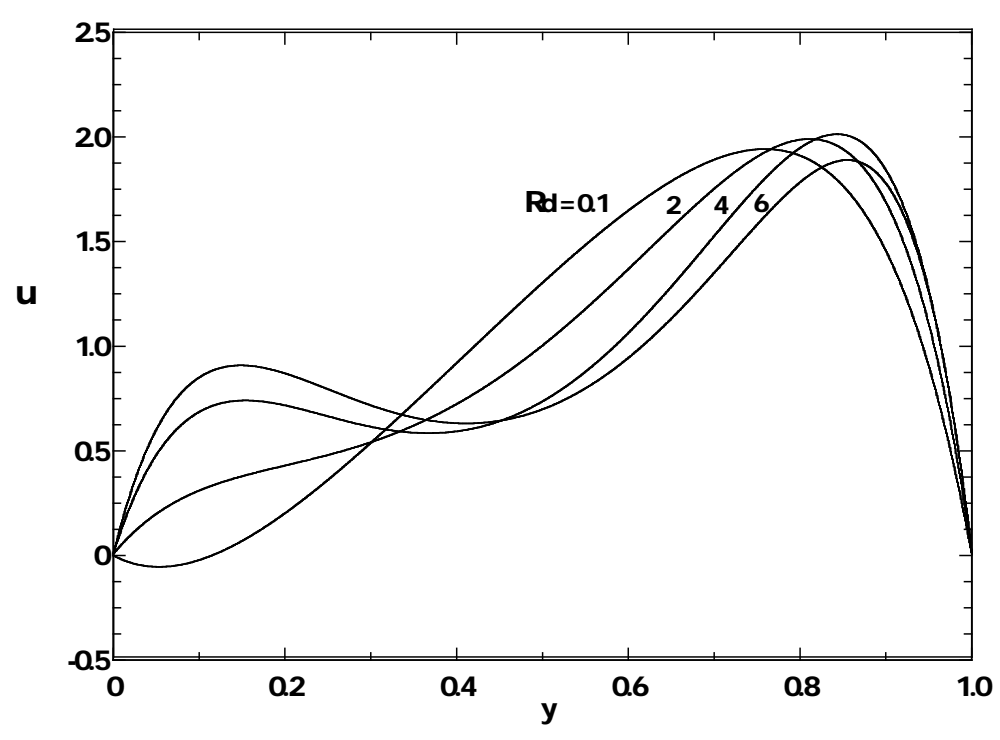

Figure 2. Effect of the radiation parameter $R_{d}$ on the velocity profile for mixed convection when $(R a / P e)=5, \theta=0.5$ for $\varphi=45^{\circ}, K^{*}=0.1, D a=0.01$.

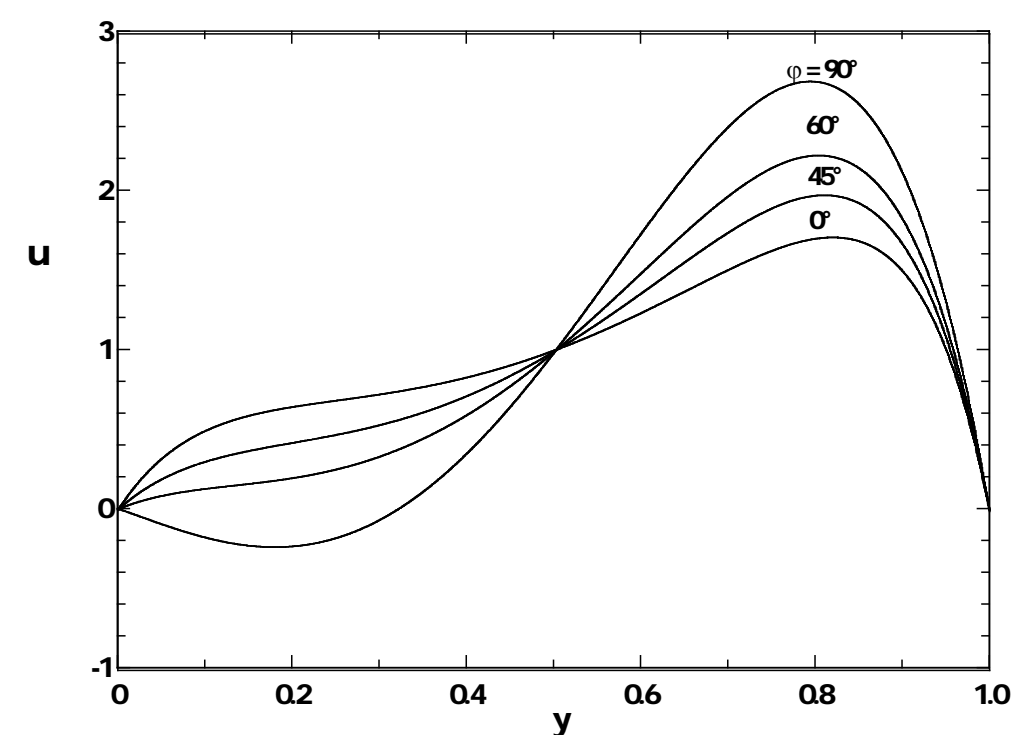

Figure 3. Effect of the inclination angle $\varphi$ on the velocity profile for mixed convection when $R_{d}=2,(R a / P e)=5, \theta=0.5$ for $K^{*}=0.1, D a=0.01$.

in the flow field when $\theta<1$. Increasing $R_{d}$ means that the release of the heat energy from the flow region and so the fluid temperature decreases.

The effects of the anisotropic parameters of the porous matrix and the thermal radiation parameter on the gradient of the pressure are presented in Figure 6 and Figure 7 as a function of Darcy number $D a$ when $(R a / P e)=10$ and $\theta=0.2$. From Figure 6 and Figure 7, it is noticed that, when $D a$ is small enough $-(\mathrm{d} P / \mathrm{d} x)$ tends asymptotically toward a constant value that depend on $K^{*}$ and $R_{d}$. The limit $D a \rightarrow 0$ corresponds to a pure Darcy medium situation for which $-(\mathrm{d} P / \mathrm{d} x)$ given by Equation (62). In pure Darcy medium, the effects of both radiation and anisotropic parameters are observed to be significant. The increase of both of them diminishes the gradient of the pressure. This affects consequently the fluid motion and contributes to reduce the reversal flow as shown in Figure 2. We concluded that radiation effect contributes to stabilize the fluid motion. For example, when $D a=10^{-3}$, from Figure 6 when $K^{*}=0.1,-(\mathrm{d} P / \mathrm{d} x) \approx 5398$ and $-(\mathrm{d} P / \mathrm{d} x) \approx 1128$ for 


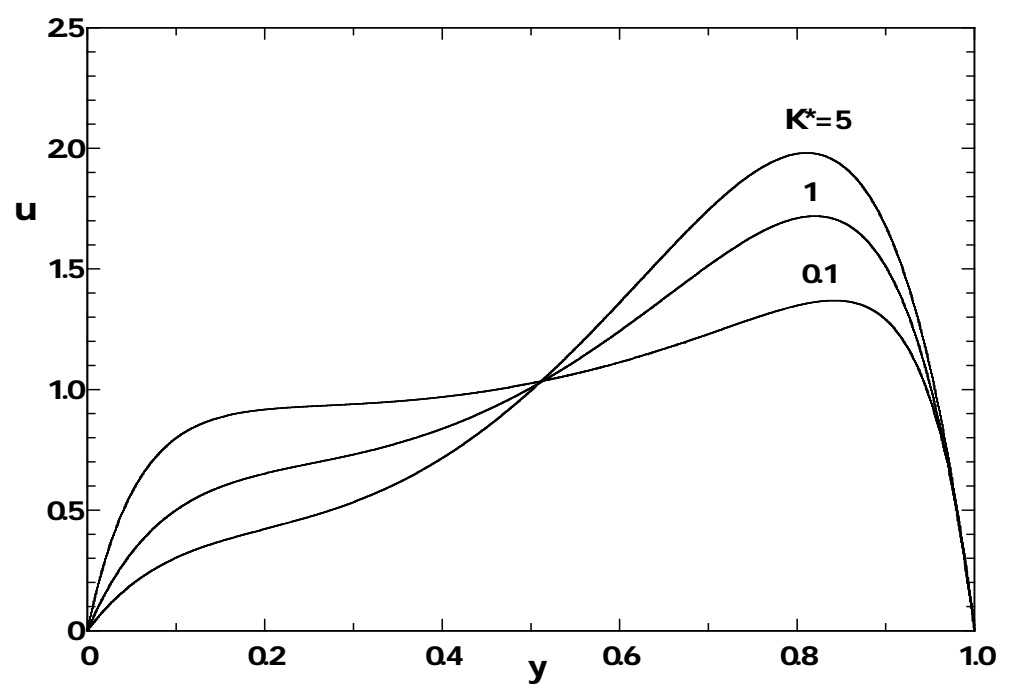

Figure 4. Effect of the permeability ratio $K^{*}$ on the velocity profile for mixed convection when $R_{d}=2,(R a / P e)=5, \theta=0.5$ for $\varphi=45^{\circ}, D a=0.01$.

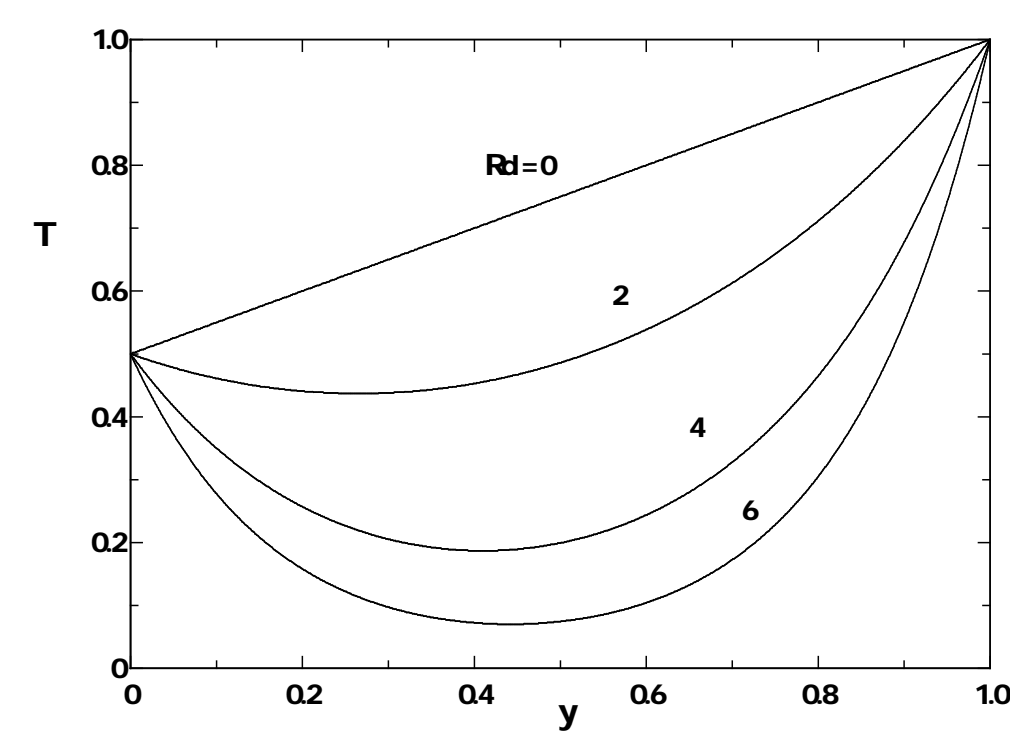

Figure 5. Temperature profile for mixed convection when $\theta=0.5$ for various values of the radiation parameter $R_{d}$.

$R_{d}=0.01$ and $R_{d}=6$ respectively. From Figure 7, for the same value of $D a$, when $R_{d}=5,-(\mathrm{d} P / \mathrm{d} x) \approx 1530$ and $-(\mathrm{d} P / \mathrm{d} x) \approx 625$ for $K^{*}=0.1$ and $K^{*}=2$ respectively. As expected from equation 43 , when the permeability of the porous medium $D a$ is increased, the boundary frictional resistance becomes gradually more important and adds to the bulk frictional drag induced by the solid matrix to slow the convection motion. As consequence, the effects of varying the values of the anisotropic parameters of the porous medium and the thermal radiation parameter become less and less important and the present solution approaches that for a pure viscous fluid indicated in Equation (42).

In Figure 8, the average wall friction $-(\overline{f R e})$ is plotted as a function of $D a$ within the porous channel when $(R a / P e)=15, \theta=0.2, K^{*}=0.1$, and $R_{d}=1$ for various values of $\varphi$. The results indicate that the average wall friction is enhanced when $\varphi$ increased. It is observed that, when $D a$ is small enough, $-(\overline{f R e})$ tends asymptotically toward a value that depends on $\varphi$ and $K^{*}$. The limit $D a \rightarrow 0$ corresponds to a pure Darcy medium for which the average wall friction variation modeled by Equation (55). In this limiting case, the effects of 


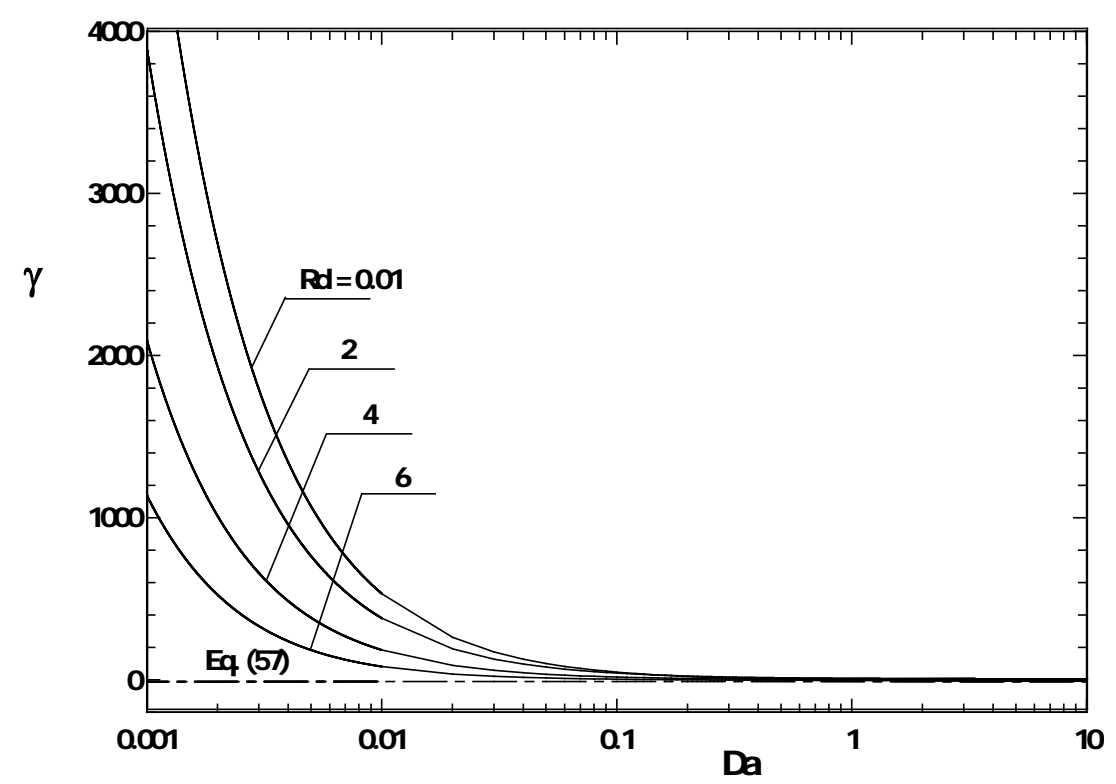

Figure 6. Effect of the Darcy number on the pressure gradient when $(R a / P e)=10$, $\theta=0.2$ for $\varphi=45^{\circ}, K^{*}=0.1$ and various values of the radiation parameter $R_{d}$.

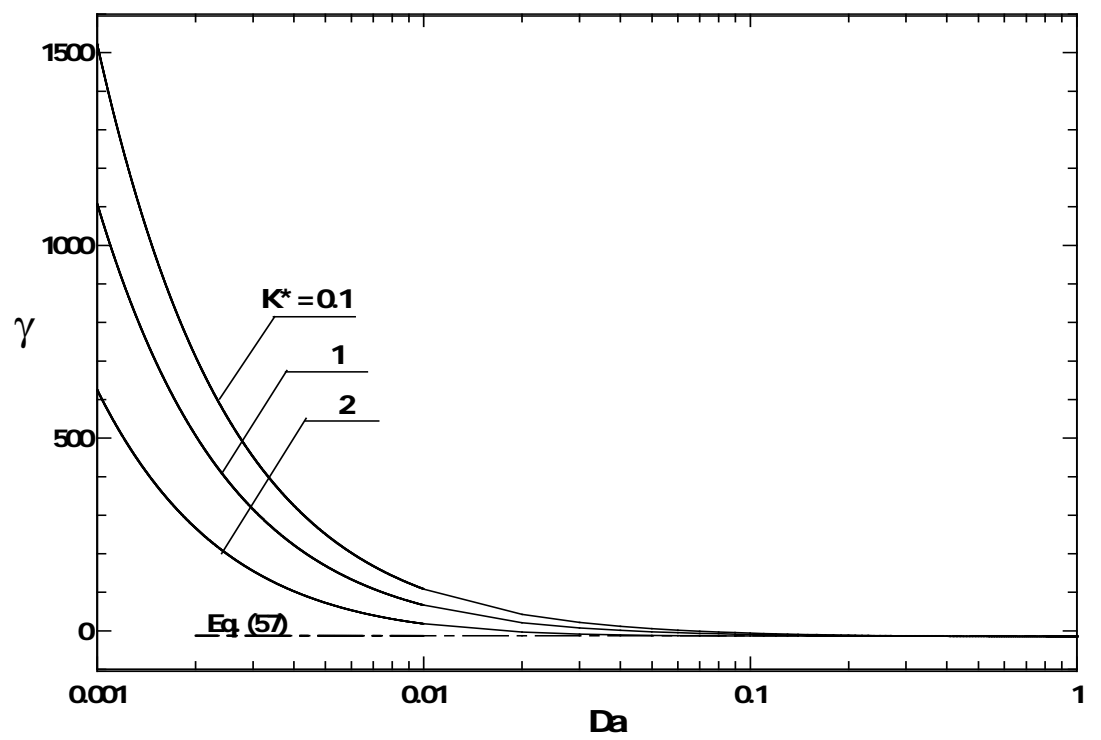

Figure 7. Effect of the Darcy number on the pressure gradient when $(R a / P e)=10$,

$\theta=0.2$ for $\varphi=45^{\circ}, R_{d}=5$ and various values of the permeability ratio $K^{*}$.

varying anisotropic parameters of the porous matrix are observed significant, since $-(\overline{f R e})$ decreases as $\varphi$ is made larger. When the Darcy number is made large enough, the results indicate that the curves, for a given value of $\varphi$, tend asymptotically toward the pure fluid situation (Equation (46)). The Darcy number required to reach this limit increases as the value of $\varphi$ is made higher. For example, this happens at $D a \approx 5$ when $\varphi=0^{\circ}$, and $D a \approx 5$ when $\varphi=90^{\circ}$.

The effects of the radiation parameter $R_{d}$ and the permeability ratio $K^{*}$ on the Nusselt number is illustrated in Figure 9 for $R a=100, D a=10^{-2}, \varphi=45^{\circ}, \theta=0.9, T_{0}=0.8$ and $\tau=0.8$. It is observed that, for a given value of $R_{d}$, the convective heat transfer increases as $R_{d}$ is made smaller. For a fixed value of $R_{d}, N u$ tends asymptotically toward a constant value as $K^{*}$ is made small enough $K^{*}<10^{-2}$. As expected from Equation (80) predicting the 


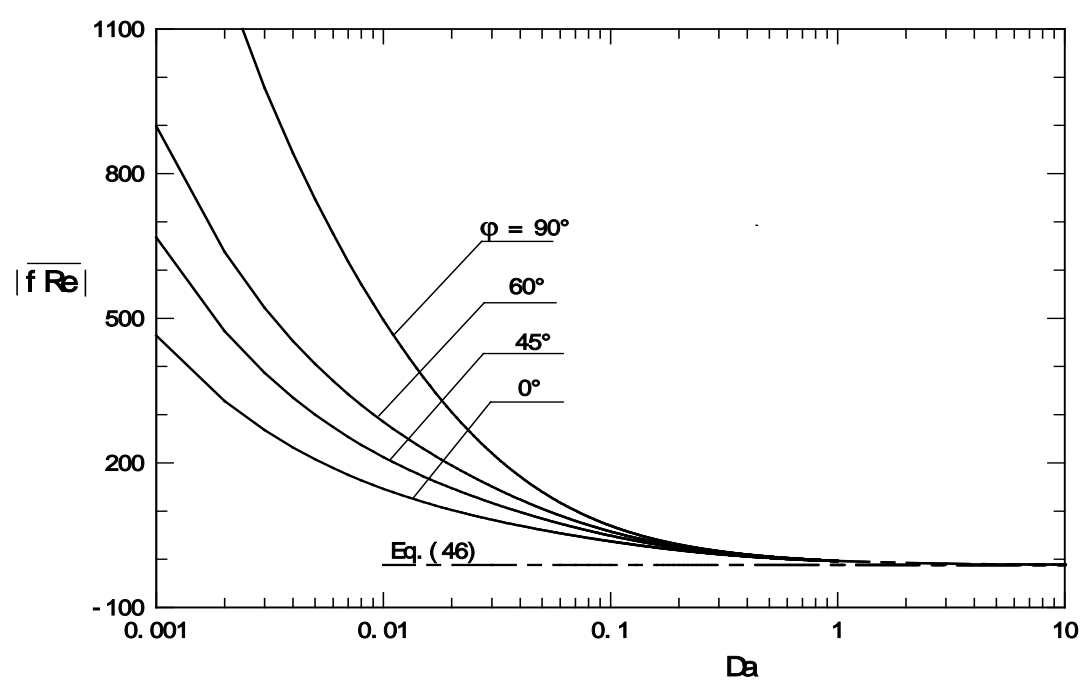

Figure 8. Effect of the Darcy number on the average wall friction for $(R a / P e)=15$, $\theta=0.2$ when $R_{d}=1, K^{*}=0.1$ and various values of the inclination angle $\varphi$.

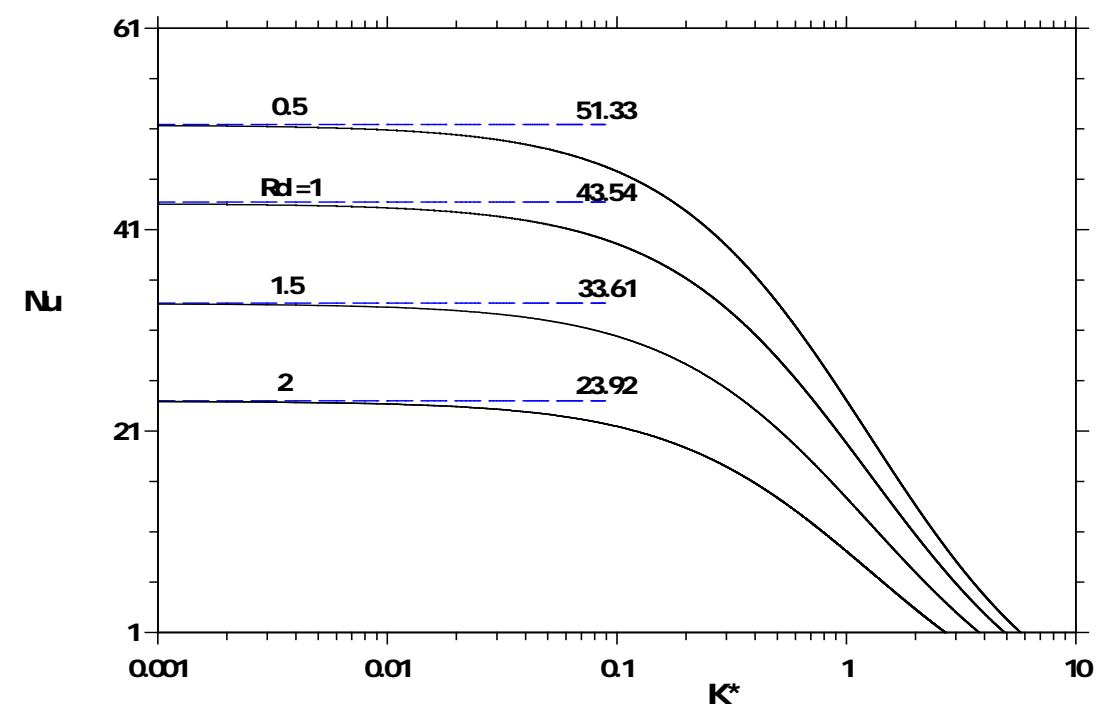

Figure 9. Effect of the permeability ratio $K^{*}$ on the Nusselt number for $R a=100$, $D a=10^{-2}, \varphi=45^{\circ}$ and $\theta=0.9, T_{0}=0.8, \tau=0.8$ and various values of $R_{d}$.

heat transfer for this limiting case, it is observed that $N u \rightarrow\left[\left(R a /\left(2 \cos ^{2} \varphi\right)\right)\left((1-\theta)^{2} / 3+\theta\right)-4(1-\theta) / 3 \tau T_{0}\right]$ when $K^{*} \rightarrow 0$. These limits are indicated as dashed lines in Figure 9 for $R_{d}=0.5,1,1.5$ and $R_{d}=2$. As it can be observed in Figure 9, the convection becomes less and less important as $K^{*}$ is made larger. Thus, for each value of $R_{d}$ considered in Figure 9, the heat transfer rate approaches the pure condition regime, $N u \rightarrow 1$ as $K^{*}$ is made large enough. The value of $K^{*}$ necessary to reach the pure condition regime depends upon $R_{d}$. For instance, for $R_{d}=0.5$, pure condition is reached when $K^{*} \approx 5.74$ while for $R_{d}=2, K^{*} \approx 3.78$.

The influence of the anisotropic orientation $\varphi$ on Nusselt number $N u$, is presented in Figure 10 where the bounding walls are submitted to various values of the radiation parameter $R_{d}$ and $K^{*}$ when $R a=100, D a=10^{-2}$, for $\theta=0.9, T_{0}=0.8, \tau=0.8$. We investigated cases where values of $R_{d}=0.5,1,1.5$ for $K^{*}=0.5,1,2$.

Curves plotted in Figure 10 reveal a symmetry of the results with respect to $\varphi=90^{\circ}$. It is observed that the heat transfer is enhanced with respect to that of an isotropic porous medium corresponding to $K^{*}=1$, when the 


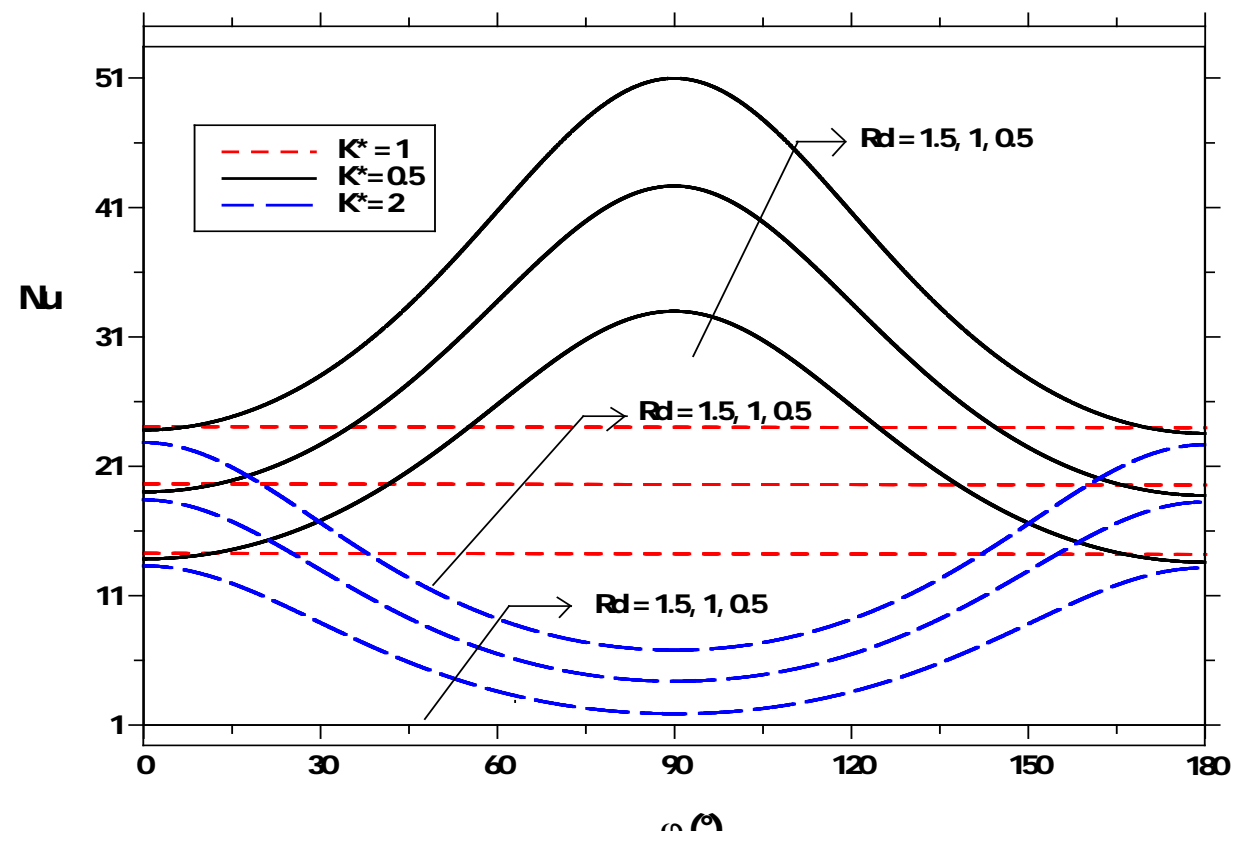

Figure 10. Effect of anisotropic angle $\varphi$ and radiation parameter $R_{d}$ on the Nusselt number for $K^{*}=0.5,1,2$ when $R a=100, D a=0.01, \theta=0.9, T_{0}=0.8$ and $\tau=0.8$.

permeability ratio $K^{*}$ is made smaller than one (i.e., $K^{*}<1$ ). Moreover, for a given $K^{*}$, the higher heat transfer rate is obtained when $R_{d}$ is smaller. For the range $0^{\circ}<\varphi<90^{\circ}$ and $K^{*}<1$, Figure 10 indicates that $\mathrm{Nu}$ is minimum at $\varphi=0^{\circ}$ for which permeability in vertical direction is minimum, but is maximum at $\varphi=90^{\circ}$. The inverse is observed for $K^{*}>1$ where the convective heat transfer is maximum at $\varphi=0^{\circ}$ and minimum at $\varphi=90^{\circ}$. For a given $R_{d}$, when $K^{*}>1\left(K^{*}<1\right), N u$ is maximum (minimum) at $\varphi=0^{\circ}$ and minimum (maximum) at $\varphi=90^{\circ}$. The maximum (minimum) increased (decreases) when $R_{d}$ is small (great) enough. Therefore, a maximum (minimum) convective heat transfer is reached when orientation of the principal axis with higher permeability of anisotropic porous medium is parallel (perpendicular) to the gravity. A Similar result has been obtained by Degan and Vasseur [17] while studying mixed convection through a vertical anisotropic porous channel with oblique principal axes.

\section{Conclusions}

A study has been made of mixed convection through a parallel-plate vertical porous channel submitted to a thermal radiative flux on its wall. The porous medium is assumed to be hydrodynamically anisotropic with its principal axes oriented in a direction that is oblique to the gravity.

Analytical expressions valid for fully developed flow and based on the generalized Brinkman-extended Darcy are obtained. The main conclusions of the present analysis are:

- Both thermal radiation and anisotropic parameters have a strong influence on the fluid motion and the heat transfer through the parallel-plate vertical porous channel.

- In the pure Darcy medium $D a \rightarrow 0$, the velocity, the average wall friction, the pressure gradient and the heat transfer rates are similar to those given by a pure Darcy analysis. In this limit, the orientation angle of the principal axes of permeability and the permeability ratio and the thermal radiation parameter affect considerably the convection heat transfer.

- In the fluid medium ( $D a>1)$, the resistance resulting from the boundary effects is predominant and adds to the bulk frictional drag induced by the solid porous matrix to slow down the convection motion. Thus, the effects of anisotropic parameters (i.e., $K^{*}$ and $\varphi$ ) and thermal radiation parameter $R_{d}$ on the convective heat transfer are progressively irrelevant, and the solutions obtained become independent of them.

- The effects of increasing values of the anisotropic parameters and the thermal radiation parameter tend to 
decrease the temperature and the gradient of the pressure. Moreover, the decrease of values of thermal radiation reduces the fluid velocity and makes the reversal flow occurs.

- For a given value of the thermal radiation parameter, a maximum (minimum) heat transfer rate through the parallel-plate vertical porous channel is obtained when the porous matrix is oriented in such a way that the principal axis with higher permeability is parallel (perpendicular) to the gravity.

\section{References}

[1] Nield, A. and Bejan, A. (1999) Convection in Porous Media. Springer, New York. http://dx.doi.org/10.1007/978-1-4757-3033-3

[2] Chen, Y.C., Chung, J.N., Wu, C.S. and Lue, Y.F. (2000) Non-Darcy Mixed Convection in a Vertical Channel Filled with a Porous Medium. International Journal of Heat and Mass Transfer, 43, 2421-2429. http://dx.doi.org/10.1016/S0017-9310(99)00299-9

[3] Mahmud, S. and Fraser, R.A. (2003) Mixed Convection-Radiation Interaction in a Vertical Porous Channel: Entropygeneration. Energy, 28, 1557-1577. http://dx.doi.org/10.1016/S0360-5442(03)00154-3

[4] Jbara, J., Slimi, K. and Mhimid, A. (2011) Combined Radiation and Natural Convection within an Open Ended Porous Channel—Validity of the Rosseland Approximation. Journal of Porous Media, 10, 862-865. http://dx.doi.org/10.1615/jpormedia.v14.i10.30

[5] Chauhan, D.S. and Rastogi, P. (2010) Radiation Effects on Natural Convection MHD Flow in a Rotating Vertical Porous Channel Partially Filled with a Porous Medium. Applied Mathematical Sciences, 13, 643-655.

[6] Slimi, K. and Nasrallah, S.B. (2004) A Numerical Study of Coupled Natural Convection and Radiation in a Porous Vertical Channel with the Finite Volume Method. Numerical Heat Transfer, 5, 451-478. http://dx.doi.org/10.1080/10407780490269003

[7] Castinel, G. and Combarnous, M. (1974) Critère d'apparition de la convection naturelle dans une couche poreuse anisotrope. Comptes Rendus Hebdomadaires des Séances de l'Académie des Sciences. Séries B, 278, 701-704.

[8] Epherre, J.F. (1975) Critère d'apparition de la convection naturelle dans une couche poreuse anisotrope. Revue Générale de Thermique, 168, 949-950.

[9] Kvernold, O. and Tyvand, P.A. (1979) Non-Linear Thermal Convection in Anisotropic Porous Media. Journal of Fluid Mechanic, 90, 609-624. http://dx.doi.org/10.1017/S0022112079002445

[10] Kimura, S., Masuda, Y. and Kazuo, H.T. (1993) Natural Convection in an Anisotropic Porous Medium Heated from the Side (Effects of Anisotropic Properties of Porous Matrix). Heat Transfer-Japanese Research, 22, 139-153.

[11] Ni, J. and Beckermann, C. (1991) Natural Convection in a Vertical Enclosure Filled with Anisotropic Porous Media. Journal of Heat Transfer, 113, 1033-1037. http://dx.doi.org/10.1115/1.2911201

[12] Tyvand, P.A. and Storesletten, L. (1991) Onset of Convection in an Anisotropic Porous Medium with Oblique Principal Axes. Journal of Fluid Mechanics, 226, 371-382. http://dx.doi.org/10.1017/S0022112091002422

[13] Zhang, X., Nguyen, T.H. and Kahawita, R. (1993) Convection Flow and Heat Transfer in an Anisotropic Porous Layer with Principal Axes Non-Coincident with the Gravity Vector. Proceeding of ASME Winter Annual Meeting, Fundamentals of Natural Convection, HTD, 264, 79-86.

[14] Zhang, X. (1993) Convective Heat Transfer in a Vertical Porous Layer with Anisotropic Permeability. Proceedings of 14th Canadian Congress of Applied Mechanics, 2, 579-580.

[15] Degan, G., Vasseur, P. and Bilgen, E. (1995) Convective Heat Transfer in a Vertical Anisotropic Porous Layer. International Journal of Heat and Mass Transfer, 38, 1975-1987. http://dx.doi.org/10.1016/0017-9310(94)00330-X

[16] Degan, G. and Vasseur, P. (1997) Boundary-Layer Regime in a Vertical Porous Layer with Anisotropic Permeability and Boundary Effects. International Journal of Heat and Fluid Flow, 18, 334-343. http://dx.doi.org/10.1016/S0142-727X(97)00011-8

[17] Degan, G. and Vasseur, P. (2002) Aiding Mixed Convection through a Vertical Anisotropic Porous Channel with Oblique Axes. International Journal of Engineering Sciences, 40, 193-209. http://dx.doi.org/10.1016/S0020-7225(01)00012-X

[18] Bejan, A. (1996) Entropy Generation Minimization. CRC Press, New York.

[19] Arpaci, V.S., Selamet, A. and Kao, S.H. (2000) Introduction to Heat Transfer. Prentice Hall, New York.

[20] Aung, W. and Worku, G. (1986) Theory of Fully Developed, Combined Convection including Flow Reversal. ASME Journal of Heat Transfer, 108, 485-488. http://dx.doi.org/10.1115/1.3246958

[21] Aung, W. and Worku, G. (1986) Developing Flow and Flow Reversal in a Vertical Channel with Asymmetric Wall Temperatures. ASME Journal of Heat Transfer, 108, 299-304. http://dx.doi.org/10.1115/1.3246919 